
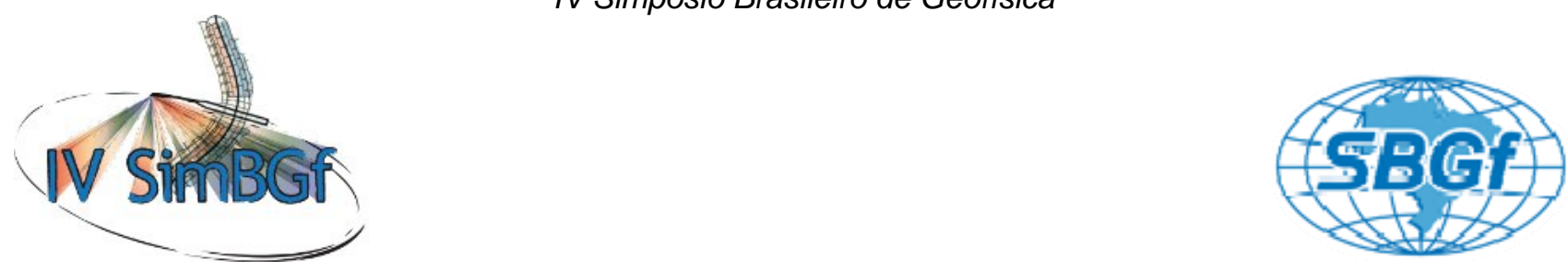

\title{
Comparação entre métodos de determinação e inversão do fator de qualidade $\mathbf{Q}$
}

Bonnie Ives de Castro Nunes ${ }^{1,2}$

Aderson Farias do Nascimento ${ }^{1,2}$

José Antônio de Morais Moreira ${ }^{2}$

Walter Eugênio de Medeiros ${ }^{1,2}$

\author{
${ }^{1}$ Programa de Pós-Graduação em Geodinâmica e Geofísica / UFRN \\ ${ }^{2}$ Departamento de Geofísica / UFRN \\ Copyright 2010, SBGf - Sociedade Brasileira de Geofísica
}

Este texto foi preparado para a apresentação no IV Simpósio Brasileiro de Geofísica, Brasília, 14 a 17 de novembro de 2010. Seu conteúdo foi revisado pelo Comitê Técnico do IV SimBGf, mas não necessariamente representa a opinião da SBGf ou de seus associados. É proibida a reprodução total ou parcial deste material para propósitos comerciais sem prévia autorização da SBGf.

\section{Resumo}

Os estudos da atenuação das ondas sísmicas é uma ferramenta importante na discriminação de reservatórios. Os processos associados são sintetizados numa descrição quantitativa chamada fator de qualidade $\mathrm{Q}$. Neste trabalho fazemos uma comparação entre diferentes métodos para determinar $\mathrm{Q}$, sendo dividido em duas partes. $\mathrm{Na}$ primeira fizemos um teste de performance de três métodos (deslocamento de pico, de centróide e razão espectral) em um modelo de três camadas. Na segunda parte invertemos os valores de $\mathrm{Q}$ utilizando os métodos de deslocamento de centróide e de pico. O deslocamento de centróides mostrou-se o mais robusto dos métodos em ambas as partes.

\section{Introdução}

Os modelos mais simples de propagação de uma onda elástica consideram o meio como ideal, mas na realidade, fatores intrínsecos do próprio meio contribuem para a perda de energia da onda. Dessa forma o pulso além de perder amplitude, sofre perda de componentes de freqüências mais altas. Este efeito denominamos de atenuação.

A atenuação de uma onda ao se propagar em um meio dispersivo tem se tornado uma ferramenta de grande importância no processamento de dados sísmicos e de GPR (Moreira, 2006). Segundo Tonn (1991), esta importância se dá por dois motivos: 1) Os efeitos da atenuação inelástica podem ser eliminados através de filtros inversos e 2) a atenuação pode ser reconhecida como um atributo sísmico significante melhorando a interpretação de sismogramas no que diz respeito à petrofísica, litologia, porosidade, permeabilidade, viscosidade e saturação das rochas (Wang, 2003; Parra, 2006, Li et al., 2006; Moreira, 2006; Zhang, 2008).

Existem diversos processos responsáveis pela atenuação de uma onda, por isso, com o objetivo de criarmos um modelo que considere a perda permanente da energia de uma onda, utiliza-se o fator de qualidade Q. Este fator reúne todos estes processos em um único parâmetro quantitativo. Por isso, muitos autores têm dedicado esforços em relacionar a atenuação com a caracterização de reservatórios devido ao fato de que em rochas porosas saturadas observa-se forte atenuação (Parra \& Hacket, 2002; Catagna et al., 2003; Tanner \& Treitel, 2003; Hübert et al., 2005; Chapman et al., 2005; Dvorkin \& Mavko, 2006).

Assim, o ponto-chave para diversas informações petrofísicas e litológicas pode estar na determinação do fator de qualidade Q. Podemos encontrar na literatura diversas abordagens para estimar Q, Tonn (1991), comparou dez abordagens e as dividiu em dois grupos: a) abordagens no domínio do tempo e b) abordagens no domínio da freqüência.

Nos últimos anos outras abordagens, como as sugeridas por Quan \& Harris (1997), Zhang \& Ulrych (2002), Rickett (2006) e Moreira (2006) têm surgido e demonstraram-se muito eficazes e robustas. Visto que hé uma grande distância temporal entre o trabalho de Tonn (1991) e os dias atuais, este trabalho tem como objetivo realizar um teste de sensibilidade entre três abordagens. São elas: razão espectral (Spencer et al., 1982), deslocamento de centróide (Quan \& Harris, 1996) e Deslocamento de pico (Zhang \& Ulrych, 2002).

Logo após realizado o teste de sensibilidade, escolhemos duas abordagens para a realização da inversão em modelos mais elaborados.

\section{Metodologia/ Problema Investigado}

O conhecimento de Q tem grande importância no uso dos filtros inversos. Monitorar a atenuação numa seção sísmica pode garantir bons resultados nas estimativas de Q. Dessa forma, Zhang \& Ulrich (2002) desenvolveram um método para determinar o fator de qualidade monitorando o deslocamento do pico do espectro de freqüência num modelo de uma camada plana (figura 1):

$$
Q=\frac{\pi t \sigma^{2}}{f_{d}-f_{p}} \text { (1) }
$$

sendo; $f_{d}$ e $f_{p}$ os picos de freqüência do sinal de entrada e do refletido na base da camada respectivamente; $t o$ tempo duplo de trânsito da chegada da reflexão e $\sigma$ o desvio padrão do espectro do sinal refletido. Quan \& Harris (1996) monitoram o deslocamento do espectro através da comparação entre centróides de freqüência (figura 2):

$$
Q=-C \pi\left(\frac{f_{c 2}-f_{c 1}}{t_{2}-t_{1}}\right)^{-1}
$$


sendo $f_{c 1}$ e $f_{c 2}$ os centróides de freqüência do primeiro e do segundo sinal registrados nos tempos $t_{1}$ e $t_{2}$ respectivamente, e $C$ a variância do espectro do segundo sinal. Spencer et al. (1982) desenvolveram uma técnica que compara o logaritmo da razão entre dois espectros (figura 3):

$$
\log \left[\frac{A_{1}(\omega)}{A_{0}(\omega)}\right]=\beta-\frac{\omega \Delta \tau}{2 Q} \text { (3) }
$$

sendo $A_{1}(\omega)$ e $A_{0}(\omega)$ os espectros de amplitudes dos sinais refletidos na base da camada e o de entrada respectivamente. Moreira (2006) inverte o fator de qualidade em múltiplas camadas comparando o deslocamento entre centróides. Nunes (2010) modifica o algoritmo comparando o deslocamento entre os picos. Os espectros são obtidos do modelo de dispersão de Futterman (1962) e como otimizador da função objeto utilizamos o método de busca iterativa COMPLEX (Richardson \& Kuester, 1973).

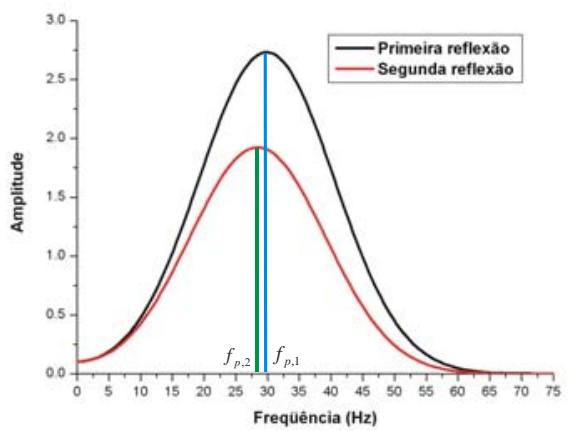

Figura 1 - Espectros de duas reflexões de chegadas em instantes diferentes. Em preto, temos a primeira reflexão. Em vermelho, temos a segunda reflexão, onde $f_{p, 1}$ e $f_{p, 2}$ são os picos de freqüência da primeira e da segunda reflexão respectivamente.

A comparação entre os métodos foi realizada utilizando um modelo de quatro camadas, sendo a última um semiespaço, em três situações distintas:

- $\quad$ Situação 1: Variamos a espessura da camada 2 e mantemos Q dessa camada constante;

- $\quad$ Situação 2: Variamos Q da camada 2 e mantemos sua espessura constante;

- Situação 3: variamos o mergulho da base da segunda camada.

Em todas as situações, a espessura e o valor de $\mathrm{Q}$ das camadas 1 e 3 são mantidas constantes. Em todos os casos contaminamos o dado com um ruído equivalente à $0,5 \%$ da amplitude da primeira reflexão.

Logo após o teste de sensibilidade escolhemos os métodos de pico e de centróide para realizarmos a inversão em um modelo de sete camadas planoparalelas.

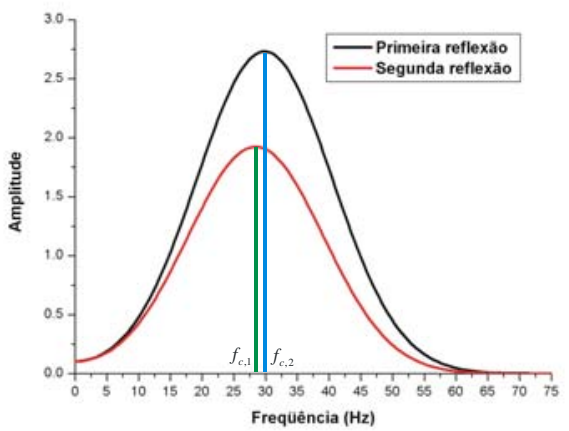

Figura 2 - Espectros de duas reflexões de chegadas em instantes diferentes. Em preto, temos a primeira reflexão. Em vermelho, temos a segunda reflexão, onde $f_{c, 1}$ e $f_{c, 2}$ são os centróides de freqüência da primeira e da segunda reflexão respectivamente.

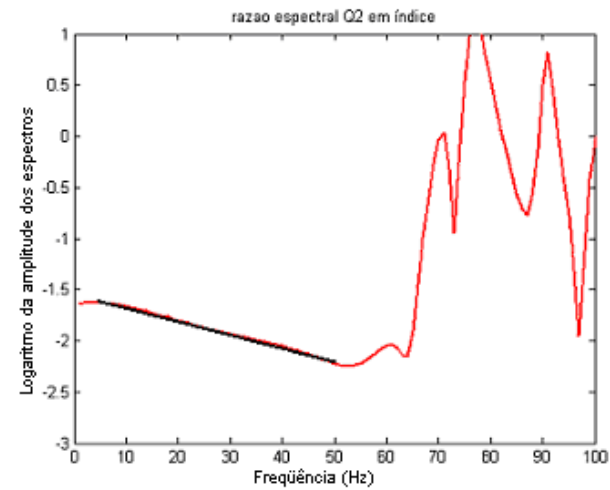

Figura 3 - Gráfico da razão espectral. No eixo horizontal, temos os valores das freqüências. No eixo vertical, temos os valores dos logaritmos das razões entre as amplitudes. $O$ fator de qualidade $Q$ das duas camadas em questão eram iguais a 50. A linha escura representa a região linear do gráfico.

\section{Resultados}

Na situação 1, para a determinação de Q da camada 2 todos os métodos apresentaram bons resultados quando se utilizaram pulsos de freqüências centrais de 30 e $40 \mathrm{~Hz}$. Os erros observados se encontravam na faixa dos $\pm 5,0 \%$ para todos os métodos. Porém, na freqüência de $60 \mathrm{~Hz}$, observamos que os métodos tornavam-se cada vez mais imprecisos à medida que aumentávamos a espessura da camada 2. O método da razão espectral demonstrou-se mais preciso nas situações 1 . No entanto, o método de deslocamento de centróides apresentou bons resultados em espessuras maiores (figuras 4, 5 e 6 ).

Ainda na situação 1 , para a determinação de $\mathrm{Q}$ da camada 3 observamos também que os métodos tornavam-se mais imprecisos com o aumento da freqüência central do pulso de entrada $\mathrm{Em}$ todas as 
freqüências utilizadas, o método de picos demonstrou-se bastante impreciso com erros atingindo mais de $25 \%$. O método de centróides foi o que mais se destacou, demonstrando bastante robustez nas freqüências centrais de $30 \mathrm{~Hz}$ e $40 \mathrm{~Hz}$. Porém, demonstrou bastante instabilidade na freqüência de $60 \mathrm{~Hz}$. O método da razão espectral também se demonstrou bastante robusto nas freqüências de $30 \mathrm{~Hz}$ e $40 \mathrm{~Hz}$, porém, perdia a instabilidade em espessuras menores da camada 2 em relação ao método de centróides (figuras 7, 8 e 9).

$\mathrm{Na}$ situação 2, mantivemos as espessuras das camadas constantes e variamos o fator de qualidade da segunda camada. Na determinação de $\mathrm{Q}$ da camada 2, o método de deslocamento de picos demonstrou bastante robustez quando utilizado o pulso de freqüência central $30 \mathrm{~Hz}$ e 40 $\mathrm{Hz}$. Porém, na freqüência de $60 \mathrm{~Hz}$, observamos uma grande instabilidade da parte do método. Os métodos de deslocamento de centróides e da razão espectral demonstraram bastante robustez para todas as freqüências centrais utilizadas sendo os que mais se destacaram nessa situação (figuras 10, 11 e 12).

Para a determinação de $\mathrm{Q}$ na camada 3 , o método de deslocamento de picos demonstrava precisão à medida que diminuíamos a atenuação da camada 2, nas freqüências de $30 \mathrm{~Hz}$ e $40 \mathrm{~Hz}$. Na freqüência central de $60 \mathrm{~Hz}$, o método demonstrou bastante instabilidade no intervalo de $\mathrm{Q}$ entre 10 e 100. Os métodos de deslocamento de centróides e o da razão espectral destacaram-se nesta situação, porém, o do deslocamento de centróides foi o mais preciso (figuras 13, 14 e 15).

Na situação 3 mantivemos os valores de Q constante em todas as camadas, porém, variamos a inclinação da interface entre as camadas 2 e 3 . O objetivo deste teste era o de observar a robustez em determinar o fator de qualidade da camada 3. Nesta situação, pudemos observar que todos os métodos foram bastante instáveis nas freqüências centrais de $30 \mathrm{~Hz}$ e $40 \mathrm{~Hz}$. Porém, quando utilizamos o pulso de freqüência central $60 \mathrm{~Hz}$, destacamos os métodos de deslocamento de centróides e o da razão espectral. O método de deslocamento de centróides foi bastante preciso até a inclinação de $16^{\circ}$, enquanto que a razão espectral manteve sua precisão até a inclinação de $12^{\circ}$ (figuras 16, 17 e 18).

Com o uso do algoritmo de inversão, o método que apresentou os melhores resultados foi o que compara os centróides. As tabelas 1 e 2 apresentam os resultados da inversão para o método de centróides e de picos respectivamente.

A grande vantagem do uso da estimativa por picos surge de sua rapidez computacional, visto que em alguns casos, este método convergia com até 9 vezes menos iterações através do método COMPLEX que o método de centróides.

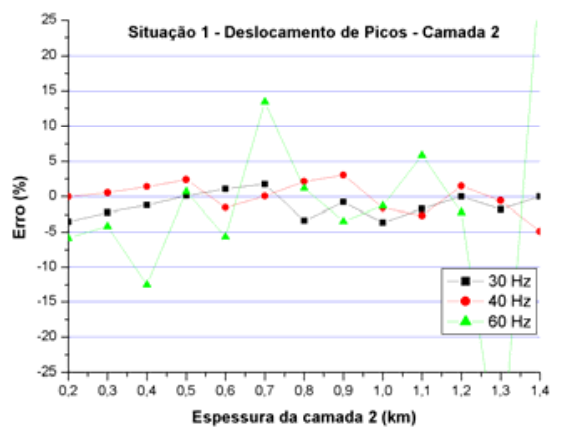

Figura 4 - Erros percentuais nas estimativas de $Q$ da camada 2 através do método de deslocamento de picos na situação 1.

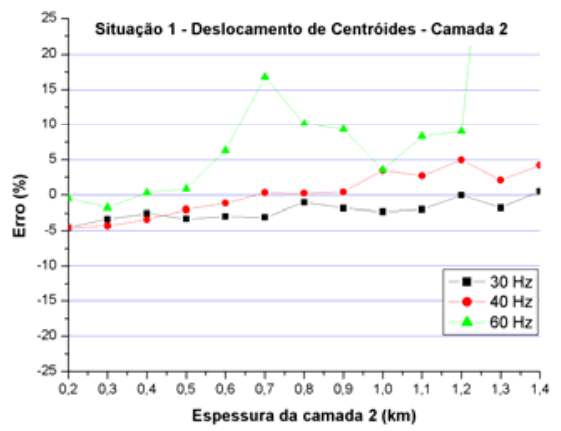

Figura 5 - Erros percentuais nas estimativas de $Q$ da camada 2 através do método de deslocamento de centróides na situação 1.

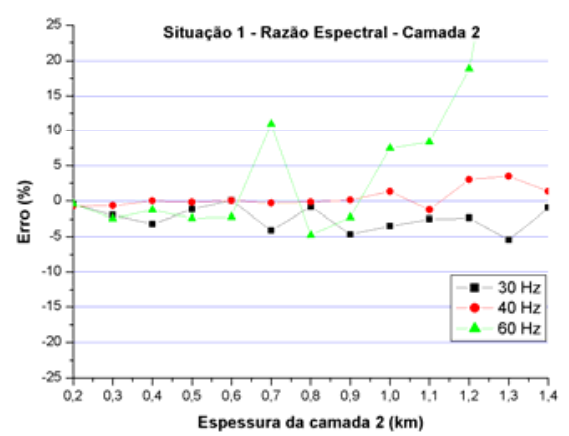

Figura 6 - Erros percentuais nas estimativas de $Q$ da camada 2 através do método razão espectral na situação 1. 


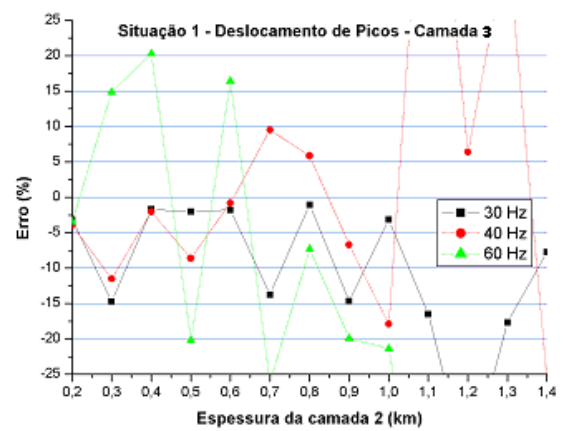

Figura 7 - Erros percentuais nas estimativas de $Q$ da camada 3 através do método de deslocamento de picos na situação 1.

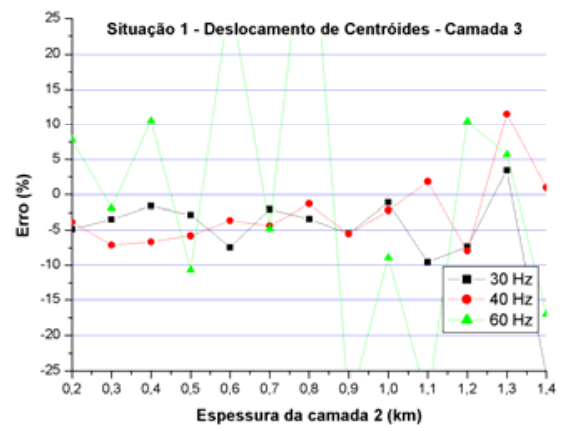

Figura 8 - Erros percentuais nas estimativas de $Q$ da camada 3 através do método de deslocamento de centróides na situação 1.

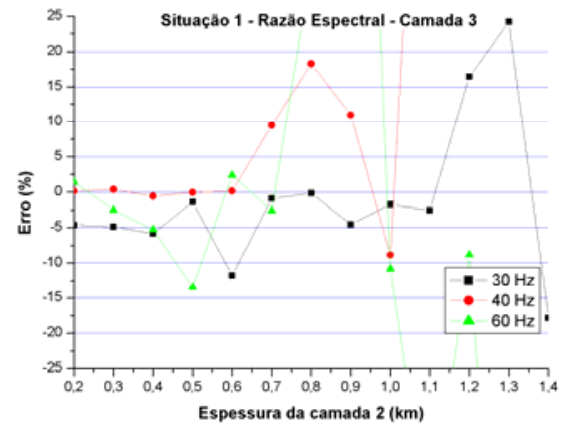

Figura 9 - Erros percentuais nas estimativas de $Q$ da camada 3 através do método razão espectral na situação 1.

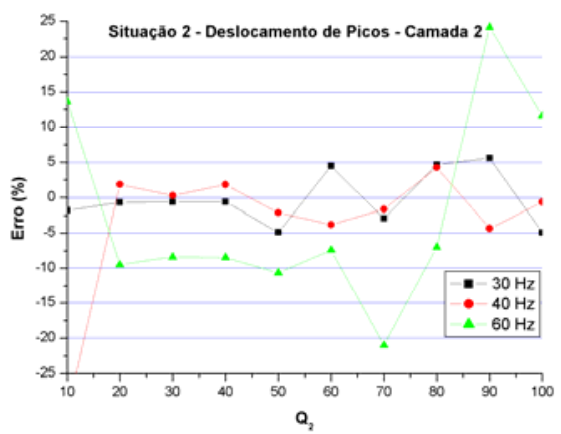

Figura 10 - Erros percentuais nas estimativas de $\mathbf{Q}$ da camada 2 através do método de deslocamento de picos na situação 2.

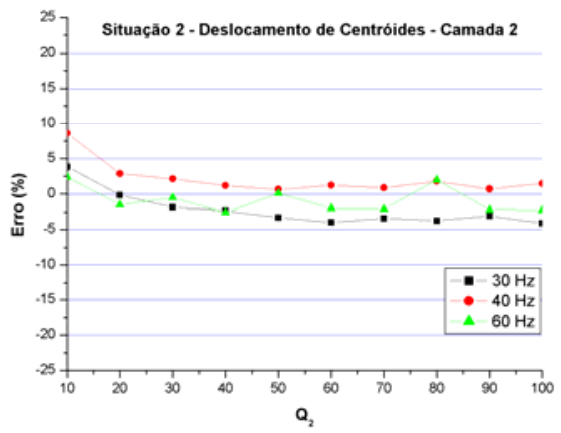

Figura 11 - Erros percentuais nas estimativas de Q da camada 2 através do método de deslocamento de centróides na situação 2.

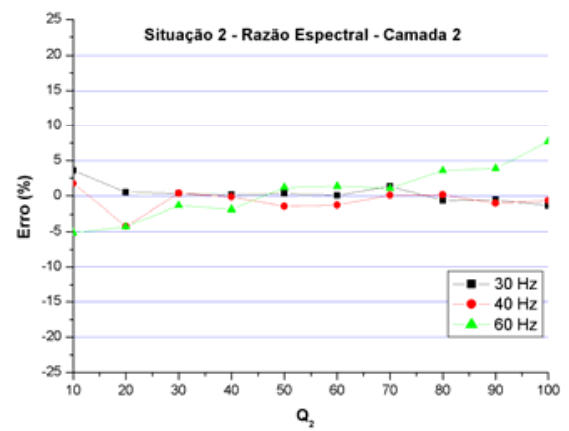

Figura 12 - Erros percentuais nas estimativas de $Q$ da camada 2 através do método de razão espectral na situação 2. 


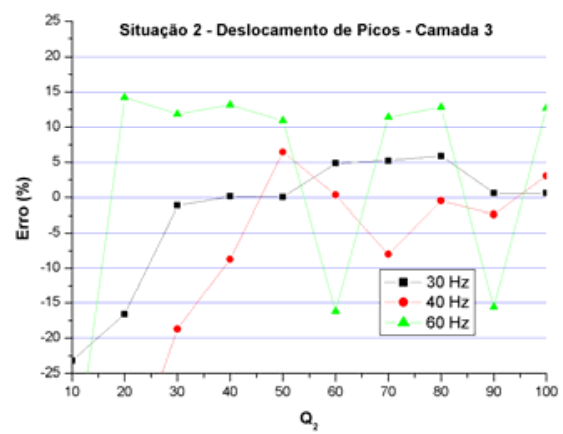

Figura 13 - Erros percentuais nas estimativas de Q da camada 3 através do método de deslocamento de picos na situação 2.

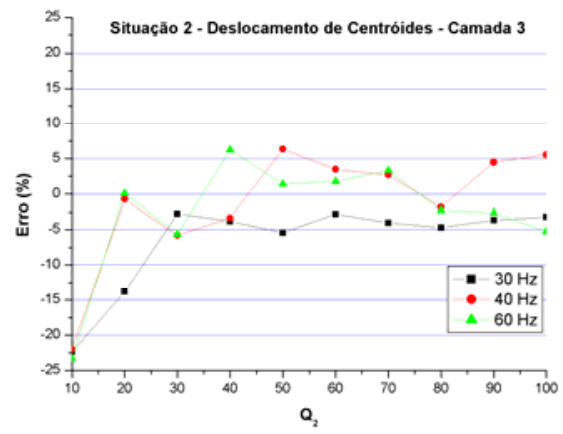

Figura 14 - Erros percentuais nas estimativas de Q da camada 3 através do método de deslocamento de centróides na situação 2 .

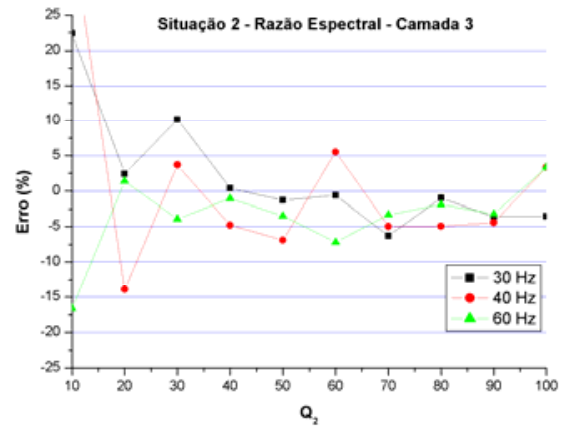

Figura 15 - Erros percentuais nas estimativas de $\mathbf{Q}$ da camada 3 através do método razão espectral na situação 2.

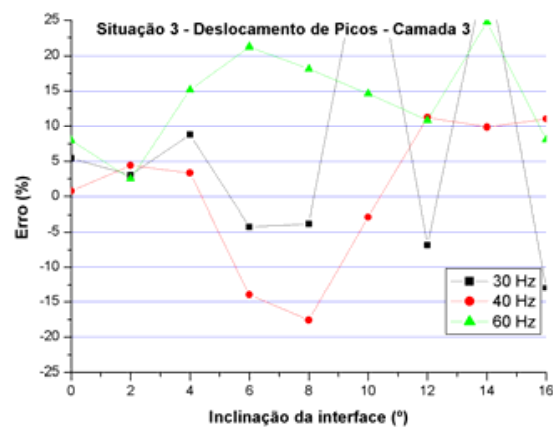

Figura 16 - Erros percentuais na estimativa de $Q$ da camada 3 para o método de deslocamento de picos na situação 3.

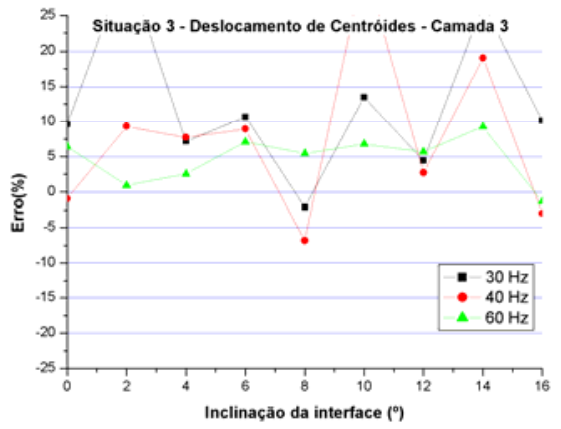

Figura 17 - Erros percentuais na estimativa de $Q$ da camada 3 para o método de deslocamento de centróides na situação 3 .

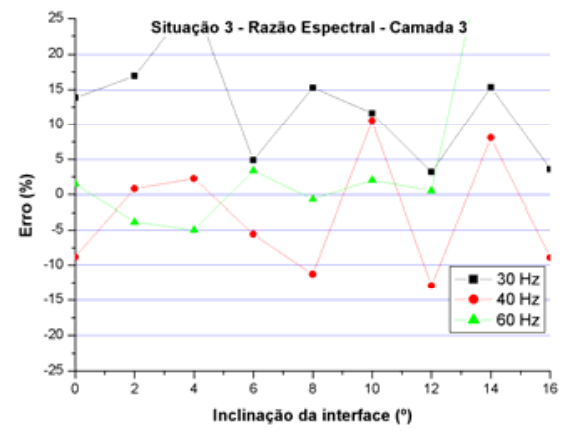

Figura 18 - Erros percentuais na estimativa de $Q$ da camada 3 para o método razão espectral na situação 3. 
Tabela 1 - Resultados da inversão através do método de comparação entre centróides.

\begin{tabular}{|c|c|c|c|c|c|c|c|}
\hline \multicolumn{9}{|c|}{ Estimativas através da inversão do modelo 1 - Centróide } \\
\hline \multicolumn{3}{|c|}{ Situação 1 } & \multicolumn{4}{|c|}{ Situação 2 } \\
\hline $\mathrm{Q}^{2}$ real & Estimativa & Erro & \multicolumn{2}{|c|}{ Q real } & Estimativa & Erro \\
\hline $\mathrm{Q}_{2}$ & 20 & 18.40 & $-8.00 \%$ & $\mathrm{Q}_{2}$ & 120 & 107.24 & $-10.63 \%$ \\
\hline $\mathrm{Q}_{3}$ & 30 & 34.19 & $13.98 \%$ & $\mathrm{Q}_{3}$ & 30 & 30.62 & $2.07 \%$ \\
\hline $\mathrm{Q}_{4}$ & 40 & 40.27 & $0.66 \%$ & $\mathrm{Q}_{4}$ & 40 & 37.79 & $-5.53 \%$ \\
\hline $\mathrm{Q}_{5}$ & 50 & 78.88 & $57.76 \%$ & $\mathrm{Q}_{5}$ & 50 & 54.53 & $9.06 \%$ \\
\hline $\mathrm{Q}_{6}$ & 40 & 34.82 & $-12.94 \%$ & $\mathrm{Q}_{6}$ & 40 & 37.78 & $-5.55 \%$ \\
\hline $\mathrm{Q}_{7}$ & 120 & 142.11 & $18.42 \%$ & $\mathrm{Q}_{7}$ & 120 & 119.66 & $-0.28 \%$ \\
\hline Iterações & \multicolumn{3}{|c|}{6186} & Iterações & \multicolumn{5}{c|}{2597} \\
\hline
\end{tabular}

Tabela 2 - Resultados da inversão através do método de comparação entre picos.

\begin{tabular}{|c|c|c|c|c|c|c|c|}
\hline \multicolumn{9}{|c|}{ Estimativas através da inversão do modelo 1 - Pico } \\
\hline \multicolumn{3}{|c|}{ Situação 1 } & \multicolumn{4}{c|}{ Situação 2 } \\
\hline $\mathrm{Q}^{2}$ real & Estimativa & Erro & \multicolumn{2}{|c|}{ Q real } & Estimativa & Erro \\
\hline $\mathrm{Q}_{2}$ & 20 & 17.84 & $-10.80 \%$ & $\mathrm{Q}_{2}$ & 120 & 130.47 & $8.72 \%$ \\
\hline $\mathrm{Q}_{3}$ & 30 & 27.5 & $-8.33 \%$ & $\mathrm{Q}_{3}$ & 30 & 32.91 & $9.69 \%$ \\
\hline $\mathrm{Q}_{4}$ & 40 & 43.9 & $9.75 \%$ & $\mathrm{Q}_{4}$ & 40 & 39.11 & $-2.23 \%$ \\
\hline $\mathrm{Q}_{5}$ & 50 & 81.56 & $63.12 \%$ & $\mathrm{Q}_{5}$ & 50 & 41.39 & $-17.22 \%$ \\
\hline $\mathrm{Q}_{6}$ & 40 & 31.47 & $-21.33 \%$ & $\mathrm{Q}_{6}$ & 40 & 32.27 & $-19.34 \%$ \\
\hline $\mathrm{Q}_{7}$ & 120 & 143.36 & $19.47 \%$ & $\mathrm{Q}_{7}$ & 120 & 119.35 & $-0.54 \%$ \\
\hline Iterações & \multicolumn{3}{|c|}{684} & Iterações $^{|c|} 589$ \\
\hline
\end{tabular}

\section{Discussão e Conclusões}

As metodologias demonstraram uma grande robustez por parte da comparação entre os centróides e da razão espectral nas freqüências centrais iguais a $30 \mathrm{~Hz}$ e 40 $\mathrm{Hz}$. O método de picos demonstrou bastante dependência com a digitalização do dado. Notamos também que, para estimarmos precisamente o fator de qualidade pelos métodos de centróide e de pico, devemos provocar uma separação considerável entre os espectros observados de duas reflexões consecutivas, desde que o sinal observado tenha uma relativa qualidade.

Quando tratamos das camadas subjacentes, o método de centróides demonstrou ser o mais eficaz mesmo quando a espessura da camada acima é relativamente grande. $O$ método da razão espectral também demonstrou uma relativa precisão, porém tornava-se menos estável antes que o método de centróides.

Nos testes onde variamos o mergulho da interface entre a segunda e a terceira camada todos os métodos demonstraram pouca precisão nas freqüências centrais de $30 \mathrm{~Hz}$ e $40 \mathrm{~Hz}$. Porém, ao aumentarmos essa freqüência para $60 \mathrm{~Hz}$ as estimativas tornavam-se mais precisas, com destaque para o deslocamento de centróides, que apresentou relativa precisão até a inclinação de $16^{\circ}$.

$\mathrm{Na}$ inversão, o algoritmo aplicado à comparação entre os centróides de freqüência tem maior robustez que o aplicado para comparar os picos. Em todos os casos, o uso do pico demonstra grande dependência com a digitalização do sinal e, mesmo sendo relativamente menos preciso que o método de centróides, é mais rápido computacionalmente utilizando 0 algoritmo COMPLEX (Richardson \& Kuester, 1973). Ambos os métodos apresentaram resultados mais precisos com a melhora da qualidade do sinal.

\section{Agradecimentos}

Agradecemos ao projeto "Inversão de fator de qualidade de dados sísmicos e aplicação em reservatórios delgados" (CNPq Proc. No. 482841/2007-9), coordenado pelo prof. Dr. Walter Eugênio de Medeiros, pelo apoio financeiro à este trabalho.

\section{Referências}

Castagna, J. P., Sun, S., and Siegfried, R. W., (2003). Instantaneous spectral analysis: Detection of lowfrequency shadows associated with hydrocarbons: The Leading Edge, 22, 120-127.

Chapman, M., Liu, E., and Li, X., (2005). The influence of abnormally high reservoir attenuation on the AVO signature: The Leading Edge, 11, 1120-1125.

Futterman, W. I. (1962). Dispersive body waves. Journal of Geophysical Research, 69, 5279-5291.

Li, H., Zhao, W., Cao, H., Yao, F. \& Shao, L. (2006). Measures of scale based on the wavelet scalogram with applications to seismic attenuation. Geophysics, 71: V111-V118.

Moreira, J. A M. (2006). Determinação do fator de qualidade $\mathrm{Q}$ baseada no deslocamento do centróide de freqüência independente do campo de velocidade. Programa de Pós-Graduação em Geodinâmica e Geofísica/UFRN, Natal/RN. Tese de Doutorado. 167 pp.

Nunes, B. I. C. (2010). Estudo comparativo de abordagens de inversão do fator de qualidade Q. Programa de Pós-Graduação em Geodinâmica e Geofísica/UFRN, Natal/RN. Dissertação de Mestrado, 96 pp.

Parra, J. \& Hackert, C. L. (2006). Modeling and interpretation of $\mathrm{Q}$ logs in carbonate rock using a double porosity model and well logs. Journal of Applied Geophysics, 58: 253-262.

Quan, Y. \& Harris, J. M. (1997). Seismic attenuation tomography using the frequency shift method. Geopysics, 62: 895-905.

Richardson, J.A. \& Kuester, J.L. (1973). Algorithm 454: The Complex Method for Constrained Optimization [E4]. Communications of the ACM, 16(8): 487-489.

Spencer, T. W., J. R. Sonnad, and T. M. Butler. (1982). Seismic Q stratigraphy or dissipation: Geophysics, 47, 16-24.

Taner, M. T. and S. Treitel. (2003). A robust method for Q estimation: $73^{\text {rd }}$ SEG, Expanded Abstracts, 710-713, Soc. of Expl. Geophys.

Tonn, R. (1991). The determination of the seismic quality factor Q from VSP Data: A comparison of different computational methods. Geophysical Prospecting, 39, 127.

Zhang, C. \& Ulrych, T. J. (2002). Estimation of quality factors from CMP records. Geophysics, 67: 1542-1547.

Zhang, C. (2008). Seismic absorbtion estimation and compensation. The University of British Columbia, Vancouver, Canada. Ph.D Thesys. 97 pp. 